\title{
Carbon Suboxide as a Quasilinear Molecule with a Large Amplitude Bending Mode
}

\section{Determination of the Molecular Constants and the $v_{7}$ Potential Function}

\author{
W. H. WEBER \\ Physics Department, Research Staff, Ford Motor Company, Dearborn, Michigan 48121
}

AND

G. W. FORD

Physics Department, University of Michigan, Ann Arbor, Michigan 48104

\begin{abstract}
The model of a quasilinear molecule with a large amplitude bending mode is used to treat $\mathrm{C}_{3} \mathrm{O}_{2}$. The Hamiltonian operator, including the rotation-vibration interaction, is derived allowing only a single vibrational degree of freedom, namely, the $\nu_{7}$ mode corresponding to the bending at the central carbon atom. The $\mathrm{C}=\mathrm{C}=0$ angle is constrained to be $180^{\circ}$. With this model the rotational energy levels and, thus, the molecular constants can be computed for any $\nu_{7}$ level once the $\nu_{7}$ potential is specified. The $l$-doubling is included only for $\pi$ states. The model contains three adjustable parameters: the rotational constant in the linear configuration and two terms in the potential function, and these are determined by fitting three experimental quantities: the rotational constants in and the separation between the ground and $2 \nu^{0}$ states. The resulting $\nu_{7}$ potential has a $30.56 \mathrm{~cm}^{-1}$ barrier at $\alpha=0$ with a minimum at $\alpha=11.04^{\circ}$, where $2 \alpha$ is the angular deviation from linearity. The model gives a good fit to the $2 \nu_{7}$ Raman data and to the rotational and centrifugal distortion constants in all of the $n \nu_{i}^{l}$ states which have been analyzed. A similar analysis is applied with equal success to the states with $\nu_{4}$, the asymmetric $\mathrm{C}=\mathrm{C}$ stretch mode at $1587 \mathrm{~cm}^{-1}$, simultaneously excited with a $\nu_{7}$ mode. The potential in this case has a $56.58 \mathrm{~cm}^{-1}$ barrier at $\alpha=0$ with a minimum at $\alpha=13.02^{\circ}$.
\end{abstract}

\section{INTRODUCTION}

Carbon suboxide is known to have a linear structure with a very low-frequency bending mode $\nu_{7}$. Recent high-resolution Fourier transform (1) and tunable laser (2) spectra of $\mathrm{C}_{3} \mathrm{O}_{2}$ have provided molecular constants both for the ground state and for a number of $\nu_{7}$ excited states as well. The purpose of this work is to determine a potential function for $\nu_{i}$ based on these new measurements and to demonstrate that the molecular constants, including centrifugal distortion, can be understood in terms of a very simple model.

The nature of the potential function for the $\nu_{7}$ mode of $\mathrm{C}_{3} \mathrm{O}_{2}$ has been of considerable interest. The ab initio calculations by Sabin and Kim (3) suggest a harmonic potential with a small quartic term, which differs greatly from most of the potentials determined by fitting experimental data. These are based either on electron diffraction results $(4,5)$ 
or infrared data $(6-8)$ and usually indicate a highly anharmonic potential which is either flat-bottomed or has a barrier of $10-50 \mathrm{~cm}^{-1}$ at the linear configuration. The most reliable of these is the one determined by Carreira et al. (8), who fit their Raman and far infrared data with a quartic well having a small $14 \mathrm{~cm}^{-1}$ quadratic barrier at the linear position.

We have used the model of Hougen et al. (9) for a quasilinear molecule to calculate the energy levels in $\mathrm{C}_{3} \mathrm{O}_{2}$. This model is an extension of an earlier one by Thorson and Nakagawa (10), who first treated the problem of the quasilinear molecule. In Section II we obtain a quantum-mechanically correct Hamiltonian operator, including rotationvibration interaction, for a molecule in which only a single vibrational degree of freedom is allowed, namely, the bending at the central carbon atom. The primary motivation for using such a simple model is the fact that $\omega_{7}\left(\approx 20 \mathrm{~cm}^{-1}\right)$ is at least a factor 25 lower in frequency than any other fundamental vibration.

In Section III we discuss the numerical solution of the Schrödinger equation to determine the energy levels. We use a simple form for the $\nu_{7}$ potential function, similar to that of Carreira et al. (8), with two adjustable parameters. As a third adjustable parameter we use the rotational constant in the linear configuration. In Section IV we determine these parameters by fitting three experimental quantities: the rotational constants in and the separation between the ground and $2 \nu_{7}{ }^{0}$ states. Despite its simplicity, the model gives a good fit to the Raman data and to the rotational and centrifugal distortion constants in all of the $n \nu_{7}{ }^{l}$ states which have been analyzed. In the same section a similar analysis is applied to the hot-band shifts, rotational constants, and centrifugal distortion constants in two $\nu_{\mathrm{s}}+n \nu_{7}{ }^{l}$ states, where $\nu_{\mathrm{s}}$ is a high-frequency stretching mode. We find that substantial changes in the $\nu_{7}$ potential occur when one of these stretching modes is excited. Thus the $\nu_{7}$ potential appears to be very sensitive to the mean molecular positions. The anomalous positive signs of $B^{\prime}-B^{\prime \prime}$ observed in several parallel bands of the type $\nu_{4} 1 \leftarrow 0$ are shown to arise from the large change in the effective $\nu_{7}$ potential. If similar changes in the $\nu_{7}$ potential occur in other stretching vibrations, then alternative assignments should be considered for some of the near ir bands studied by Mantz et al. (1).

\section{THEORY}

\section{A. The Classical Kinetic Energy}

We consider a model of $\mathrm{C}_{3} \mathrm{O}_{2}$ in which the $\mathrm{C}=\mathrm{C}=\mathrm{O}$ bonds are rigid while the $\mathrm{C}=\mathrm{C}=\mathrm{C}$ bond bending is allowed (see Fig. 1). The bending angle is $2 \alpha$, and we choose a bodyfixed coordinate system oriented so the $y z$-plane is the plane of the molecule with the $z$-axis parallel to the $\mathrm{O}-\mathrm{O}$ line. The distance between the center of mass and the central carbon is $R \sin \alpha$, where

$$
R=2\left[\left(m_{\mathrm{C}} r_{\mathrm{C}}+m_{\mathrm{o}} r_{\mathrm{o}}\right) /\left(3 m_{\mathrm{C}}+2 m_{\mathrm{o}}\right)\right] .
$$

The principal moments of inertia are

$$
\begin{aligned}
I_{y y} & =I_{0} \cos ^{2} \alpha, \\
I_{z z} & =\mu \sin ^{2} \alpha, \\
I_{x x} & =I_{y y}+I_{z z}=I_{0} \cos ^{2} \alpha+\mu \sin ^{2} \alpha,
\end{aligned}
$$




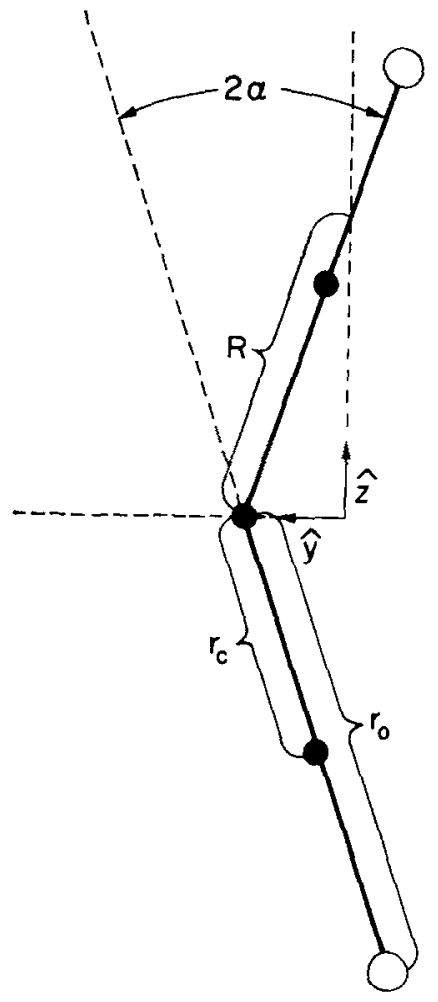

IIG. 1. Coordinate system used to describe the bending mode of $\mathrm{C}_{3} \mathrm{O}_{2}$.

where

$$
\begin{aligned}
I_{0} & =2 m_{\mathrm{C}} r_{\mathrm{C}}^{2}+2 m_{\mathrm{O}} r_{\mathrm{O}}{ }^{2}, \\
\mu & =I_{0}-\left(3 m_{\mathrm{C}}+2 m_{\mathrm{O}}\right) R^{2}
\end{aligned}
$$

are the moments of inertia in the $\alpha=0$ and $\alpha=\pi / 2$ configuration, respectively.

The coordinate of the central carbon atom with respect to the center of mass is

$$
\mathbf{r}_{1}=R \sin \alpha \hat{\mathbf{y}},
$$

the coordinates of the outer carbons are

$$
\mathbf{r}_{2,3}=\left(R-r_{\mathrm{C}}\right) \sin \alpha \hat{\mathbf{y}} \pm r_{\mathrm{C}} \cos \alpha \hat{\mathbf{z}},
$$

while the coordinates of the oxygens are

$$
\mathbf{r}_{4,5}=\left(R-r_{0}\right) \sin \alpha \hat{\mathbf{y}} \pm r_{O} \cos \alpha \hat{\mathbf{z}} .
$$

The kinetic energy for a fixed center of mass and fixed orientation of the body fixed axes is

$$
T_{\mathrm{vib}}=\frac{1}{2} m_{\mathrm{C}}\left(\dot{\mathbf{r}}_{1}^{2}+\dot{\mathbf{r}}_{2}^{2}+\dot{\mathbf{r}}_{3}^{2}\right)+\frac{1}{2} m_{\mathrm{O}}\left(\dot{\mathbf{r}}_{1}^{2}+\dot{\mathbf{r}}_{5}^{2}\right)
$$

Forming the time derivatives we find

$$
T_{\mathrm{v} \dot{ } b}=\frac{1}{2} I_{\alpha \alpha \dot{\alpha}} \dot{\alpha}^{2}
$$


where

$$
I_{\alpha \alpha}=\mu \cos ^{2} \alpha+I_{0} \sin ^{2} \alpha .
$$

The rotational kinetic energy is

$$
T_{\text {rot }}=\frac{1}{2}\left(I_{x x} \omega_{x}^{2}+I_{y y} \omega_{y}^{2}+I_{z z} \omega_{z}^{2}\right)
$$

where, in terms of the Euler angles $(\phi, \theta, \psi)(11)$,

$$
\begin{aligned}
& \omega_{x}=\sin \theta \sin \psi \dot{\phi}+\cos \psi \dot{\theta}, \\
& \omega_{y}=\sin \theta \cos \psi \phi-\sin \psi \dot{\theta}, \\
& \omega_{z}=\cos \theta \dot{\phi}+\psi,
\end{aligned}
$$

are the body-fixed components of the angular velocity. The total kinetic energy in the center of mass system is the sum of (8) and (10).

\section{B. The Hamiltonian Operator}

When the classical kinetic energy is a quadratic form in the generalized velocities, the quantum Hamiltonian operator is formed according to the following prescription. One writes

$$
T=\frac{1}{2} g_{j k} \dot{q}^{j} \dot{q}^{k},
$$

where the $q$ 's are the generalized coordinates and $g_{j k}=g_{j k}(q)$ is the mass tensor. The quantum Hamiltonian operator is then (12)

$$
H=-\left(\hbar^{2} / 2\right)\left(1 / g^{\frac{1}{2}}\right)\left(\partial / \partial q^{j}\right) g^{\frac{1}{2}} g^{j k}\left(\partial / \partial q^{k}\right)+V(q),
$$

where $V(q)$ is the potential energy. In this expression $g^{i k}$ is the inverse of the mass tensor and $g$ is its determinant. The Hamiltonian (13) is self-adjoint with respect to the scalar product

$$
\langle\phi, \chi\rangle=\int \phi^{*} \chi g^{\frac{1}{2}} d q_{1} \cdots d q_{n} .
$$

The form we use for the Hamiltonian differs from the commonly used Podolsky form, for which the factor $g^{\frac{1}{2}}$ is omitted in the scalar product (13).

From the expressions (8) and (10) we find the nonzero elements of $g_{j k}$ are

The determinant is

$$
\begin{aligned}
g_{\alpha \alpha} & =I_{\alpha \alpha}, \\
g_{\theta \theta} & =I_{y y}+I_{z z} \sin ^{2} \phi, \\
g_{\theta \psi} & =g_{\psi \theta}=-I_{z z} \sin \theta \sin \phi \cos \phi, \\
g_{\psi \psi} & =I_{y y} \sin ^{2} \theta+I_{z z}\left(\cos ^{2} \theta+\sin ^{2} \theta \cos ^{2} \phi\right), \\
g_{\theta \psi} & =g_{\psi \theta}=I_{z z} \cos \theta, \\
g_{\theta \theta} & =I_{z z} .
\end{aligned}
$$

Forming (13) we can write

$$
g=I_{\alpha \alpha} I_{x x} I_{y y} I_{z z} \sin ^{2} \theta
$$

$$
H=H_{\text {rot }}+H_{\text {vib }} \text {. }
$$


Here

$$
H_{\text {rot }}=\left(J_{x}^{2} / 2 I_{x x}\right)+\left(J_{y}^{2} / 2 I_{y y}\right)+\left(J_{z}^{2} / 2 I_{z z}\right)
$$

is the rotational IIamiltonian with $\mathbf{J}$ the total angular momentum operator, and

$$
I_{\mathrm{vib}}=-\frac{\hbar^{2}}{2} \frac{1}{\left(I_{\alpha \alpha} I_{x x} I_{y y} I_{z z}\right)^{\frac{1}{2}}} \frac{\partial}{\partial \alpha}\left(\frac{I_{x x} I_{y y} I_{z z}}{I_{\alpha \alpha}}\right)^{\frac{1}{2}} \frac{\partial}{\partial \alpha}+\mathrm{V}(\alpha)
$$

is the bending mode vibrational Hamiltonian.

\section{The Eigenvalue Problem}

We seek a solution of the eigenvalue problem

in the form

$$
H \Psi=E \Psi
$$

$$
\Psi=\sum_{l=-J}^{J} \phi_{l}(\alpha) \Phi_{J l M}(\phi, \theta, \psi),
$$

where $\Phi_{J l m}$ are the symmetric top eigenfunctions (14)

$$
\begin{aligned}
\mathbf{J}^{2} \Phi_{J l M} & =\hbar^{2} J(J+1) \Phi_{J l M}, \\
\hat{\mathbf{z}} \cdot \mathbf{J} \Phi_{J l M} & =\hbar l \Phi_{J l M}, \\
\hat{\mathbf{Z}} \cdot \mathbf{J} \Phi_{J l M} & =\hbar M \Phi_{J l M} .
\end{aligned}
$$

Here $\hat{\mathbf{Z}}$ is the space-fixed polar axis and $\hat{\mathbf{z}}$ is our body-fixed axis (Fig. 1). Our choice of $l$ (instead of $K$ ) to represent the quantum number associated with the body-fixed axis projection of the total angular momentum corresponds to the usual convention for a quasilinear molecule.

The rotational Hamiltonian (18) can be written

$$
H_{\mathrm{rut}}=\frac{I_{x x}+I_{y y}}{4 I_{x x} I_{y y}}\left(\mathbf{J}^{2}-J_{z}^{2}\right)+\frac{1}{2 I_{z z}} J_{z}{ }^{2}+\frac{I_{x x}-I_{y y}}{8 I_{x x} I_{y y}}\left(J_{+}{ }^{2}+J_{-}{ }^{2}\right) .
$$

Here $J_{-}$and $J_{+}$are the raising and lowering operators

with the properties (15)

$$
J_{ \pm}=J_{x} \pm i J_{y}
$$

$$
J_{ \pm} \Phi_{J l M}=\hbar[(J \pm l)(J \mp l+1)]^{\frac{1}{1} \Phi_{J, l \mp 1, M} .}
$$

The symmetric top eigenfunctions are eigenfunctions of the rotational Hamiltonian for the case $I_{x x}=I_{y y}$, i.e., when the last term vanishes. The corresponding energy eigenvalues are independent of $M$ and the sign of $l$. When $I_{x x} \neq I_{y y}$ and the last term in (23) is present, its effect, as we see from (25), is to mix states with $l$ values differing by two units. This removes the $|l|$ degeneracy and leads to the well-known $l$-type doubling effect (16). 
We insert the Ansatz (21) in the eigenvalue problem (20) with $H$ given by (17) and (23). Using the properties (22) and (25) of the rotational operators and the orthogonality of the $\Phi_{J l M}$, we can write the eigenvalue problem in the form of a finite set of coupled equations for $\phi_{l}(\alpha), l=-J,-J+1, \ldots, J$. That is,

$$
\left(H_{l}-E\right) \phi_{l}+U(\alpha)\left[k(J, l) \phi_{l+2}+k(J,-l) \phi_{l-2}\right]=0,
$$

where

$$
\begin{aligned}
H_{l} & =H_{\mathrm{vib}}+\frac{I_{x x}+I_{y y}}{4 I_{x x} I_{y y}} \hbar^{2}\left[J(J+1)-l^{2}\right]-\frac{\hbar^{2} l^{2}}{2 I_{z z}}, \\
U(\alpha) & =\frac{I_{x x}-I_{y y}}{8 I_{x x} I_{y y}} \hbar^{2},
\end{aligned}
$$

and

$$
k(J, l)=[(J-l-1)(J-l)(J+l+1)(J+l+2)]^{\frac{1}{2}} .
$$

Since in (26) only $l$ values which differ by 2 are coupled, the odd and even $l$ values are uncoupled. Moreover, since $H_{l}$ is even in $l$ and $k(J,-l)=k(J, l-2)$ we readily see that the symmetric and antisymmetric combinations of $\phi_{l}$ and $\phi_{-l}$ are separately coupled.

For a quasilinear molecule the bending angle $\alpha$ is always small. Using (2) we see that $U(\alpha) \propto \alpha^{2}$ and, therefore, the coupling terms in (26) will be small for such molecules. Neglecting the coupling terms gives a separate eigenvalue problem for each $l$, the energy eigenvalues being the eigenvalues of $H_{l}$. Since $H_{l}$ is even in $l$, the spectra of $H_{l}$ and $H_{-l}$ are identical; the corresponding eigenvalues are degenerate. If, now, the coupling terms are included, the only eigenstates which are directly coupled are the symmetric and antisymmetric combinations for $l= \pm 1$. We therefore approximate the set of coupled equations (26) by neglecting the coupling terms for $|l| \neq 1$ :

$$
\left(H_{l}-E\right) \phi_{l}=0, \quad|l| \neq 1 \text {. }
$$

For $|l|=1$, we write

$$
\begin{aligned}
& \phi_{1 \mathrm{~s}}=\frac{1}{2}\left(\phi_{1}+\phi_{-1}\right), \\
& \phi_{1 \mathrm{a}}=\frac{1}{2}\left(\phi_{1}-\phi_{-1}\right),
\end{aligned}
$$

and, neglecting the couplings with $l= \pm 3$, we get

and

$$
\left(H_{1}-E\right) \phi_{1 \mathrm{~s}}+J(J+1) U(\alpha) \phi_{1 \mathrm{~s}}=0,
$$

$$
\left(H_{1}-E\right) \phi_{1 \mathrm{a}}-J(J+1) U(\alpha) \phi_{1 \mathrm{a}}=0 .
$$

The equations (30), (32), and (33) are separate eigenvalue problems for second-order differential operators, whose numerical solution will be discussed in the following section. The approximations involved in obtaining these equations amount to neglecting second-order quantities in the $l$-doubling term in the rotational Hamiltonian (23). However, our approximation is not equivalent to first-order perturbation theory, since for $|l|=1$ the coupling is treated exactly. For example, this more exact treatment predicts a difference in the centrifugal distortion terms associated with the symmetric and antisymmetric $|l|=1$ states, which does not result from first-order perturbation theory. 


\section{NUMERICAL SOLUTION}

\section{A. Numerical Solution of the Eigenvalue Problem}

It will be convenient to make the change of independent variable,

$$
u=\sin ^{2} \alpha,
$$

and to introduce the dimensionless parameter

$$
\xi=\mu / I_{0}
$$

and the dimensionless energy eigenvalue

$$
\lambda=I_{0} E / 2 \hbar^{2} .
$$

Then, using (27), (19) and the definitions (2) we can write the eigenvalue problems (30), (32), and (33) in the standard form (17)

where

$$
(d / d u) p(u)(d \phi / d u)-q(u) \phi+\lambda r(u) \phi=0
$$

and

$$
\begin{aligned}
& p(u)=\left(\frac{1-u+\xi u}{u+\xi-\xi u}\right)^{\frac{1}{2}} u(1-u), \\
& r(u)=[(1-u+\xi u)(u+\xi-\xi u)]
\end{aligned}
$$

$$
\begin{aligned}
q(u)=\frac{1}{4} r(u)\left\{\frac{2 I_{0}}{h^{2}} V(u)\right. & +\frac{1-u+\frac{1}{2} \xi u}{(1-u)(1-u+\xi u)}\left[J(J+1)-l^{2}\right] \\
& \left.\quad+\frac{l^{2}}{\xi u} \pm \frac{\xi u J(J+1)}{4(1-u)(1-u+\xi u)}-\delta_{|l|, 1}\right\} .
\end{aligned}
$$

In the last expression the + sign in front of the last term gives (32), the - sign gives (33).

Equation (36) is a singular Sturm-Liouville differential equation on the interval $0<u<1$, self-adjoint with respect to the scalar product.

$$
\langle\phi, \chi\rangle=\int_{0}^{1} d u r(u) \phi^{*}(u) \chi(u) .
$$

The endpoints of the interval are regular singular points of the differential equation, which implies that for an arbitrary value of $\lambda$ there will be exactly one solution, $\phi_{+}(\lambda, u)$, which is regular at $u=0$, and one solution, $\phi_{-}(\lambda, u)$, which is regular at $u=1$. More precisely, assuming $V(u)$ is regular in the interval $0 \leq u \leq 1$, from (36) we see we can choose

and

$$
\phi_{+}(\lambda, u)=u^{\frac{2}{2}[l \mid} f_{+}(\lambda, u), \quad f_{+}(\lambda, 0)=1,
$$

with

$$
\phi_{-}(\lambda, u)=(1-u)^{\beta(J, l)} f_{-}(\lambda, u), \quad f_{-}(\lambda, 1)=1,
$$

$$
\beta=\frac{1}{4}\left[2 J(J+1)-2 l^{2} \pm J(J+1) \delta_{|l|, 1}\right]
$$

Here $f_{+}(\lambda, u)$ is analytic at $u=0$ and $f_{-}(\lambda, u)$ is analy tic at $u=1$. The eigenvalues are 
those discrete values of $\lambda$ for which (36) has solutions regular at both endpoints. Thus the eigenvalues are characterized by the requirement that the solutions $\phi_{+}(\lambda, u)$ and $\phi_{-}(\lambda, u)$ be linearly dependent. This requirement in turn is fulfilled if and only if

$$
\Delta(\lambda) \equiv p(u)\left[\phi_{-}(\lambda, u) \frac{d \phi_{+}(\lambda, u)}{d u}-\phi_{+}(\lambda, u) \frac{d \phi_{-}(\lambda, u)}{d u}\right]=0 .
$$

The quantity in square brackets is just the usual Wronskian and, since $p(u)$ is always positive within the interval, the requirement (44) is equivalent to requiring that the Wronskian vanishes. The point of introducing $\Delta(\lambda)$ is that it is independent of the point $u$, as is easily shown using the differential equation (36).

Our method of numerical solution of the eigenvalue problem (36) is straightforward. We first pick an approximate value of the eigenvalue $\lambda$. For this value of $\lambda$ we form $\phi_{+}(\lambda, u)$ by integrating numerically from $u=0$ in the $+u$ direction; likewise we form $\phi_{-}(\lambda, u)$ by integrating from $u=1$ in the $-u$ direction. Our method of integration is to write (36) as a pair of coupled first-order equations for the dependent variables $\phi$ and $\psi \equiv p(u) d \phi / d u$, and use the three-point Adams-Bashforth integration scheme (18). At an intermediate point we form

$$
\Delta(\lambda)=\phi_{-} \psi_{+}-\phi_{+} \psi_{-} .
$$

This procedure is repeated, using Newton's method for finding the zeros of a function, until a value of $\lambda$ results for which $\Delta(\lambda)$ vanishes. Although the convergence of this procedure is sensitive to the first guess, we find in general that six iterations give convergence to a part in $10^{7}$.

As a check on the numerical accuracy of this procedure we have applied it to the special case

$$
p(u)=u(1-u), \quad q(u)=0, \quad r(u)=1,
$$

for which the eigenfunctions are the Legendre polynomials $P_{n}(1-2 u)$ with eigenvalues $\lambda=-n(n+1)$. We found for $n \lesssim 8$, the numerical eigenvalues are accurate to a part in $10^{6}$, and we expect a corresponding accuracy in our solutions of (36).

The numerical procedure described above is very efficient. Typical times for the determination of each eigenvalue on the DEC-10 computer are a few seconds.

\section{B. Choosing the Polential}

In order to determine the spectrum of energy eigenvalues we must specify the potential energy function $V(u)$ and the parameters $\xi$ and

$$
B_{0} \equiv \frac{\hbar}{4 \pi c I_{0}}
$$

which is the rotational constant (in wavenumbers) for the linear configuration of the molecule. A simple form of the potential which allows a barrier in the linear configuration and a significant anharmonicity is given by

$$
V(u)=V_{2} u+V_{4} u^{2}=V_{2} \sin ^{2} \alpha+V_{4} \sin ^{4} \alpha,
$$

where $V_{2}$ and $V_{4}$ will be in wavenumbers. We have chosen to keep the parameter $\xi$ 
constrained at the value

$$
\xi=0.2423
$$

which is determined from (34) and (3) using the atomic separations $r_{0}=2.4544 \AA$, $r_{C}=1.2899 \AA$, measured by electron diffraction $(4,5)$. The parameter $B_{0}$ determined by the same method is $0.0725 \mathrm{~cm}^{-1}$. This value, however, is not accurate enough, since we will be attempting to fit precise infrared measurements of the rotational constants. Thus we treat $B_{0}, V_{2}$, and $V_{4}$ as the independent parameters with which we fit the experimental data.

\section{COMPARISONS WITH EXPERIMENTAL RESULTS}

\section{Only $\nu_{7}$ Modes Excited}

In this section we compare the theoretical and experimental results for those levels in which only the low-frequency bending mode $\nu_{7}$ is excited. The three adjustable parameters needed in the theoretical calculations can be determined by fitting three appropriate experimental quantities exactly or by fitting in a least-squares manner some larger set of data. We have chosen to fit exactly the $B$ values in the ground and $2 \nu_{7}^{\prime \prime}$ states and the energy separation between them reported in Ref. (1). Each of these quantities has been measured by at least one other independent experiment, and the accuracy with which they are known is very good compared with other possible data, e.g., Raman or far-infrared measurements.

The parameters which result from the above fitting procedure are $B_{0}=0.0735138$ $\pm 8 \times 10^{-6} \mathrm{~cm}^{-1}, V_{2}=-1666 \pm 17 \mathrm{~cm}^{-1}$, and $V_{4}=22702 \pm 120 \mathrm{~cm}^{-1}$. The uncertainties result primarily from the uncertainty in the $2 \nu_{7}{ }^{0}$-state $B$-value. This potential energy function, which is very similar to the one determined by Carreira et al. (8), has a $30.56 \mathrm{~cm}^{-1}$ barrier at the linear configuration with a minumim at $\alpha=11.04^{\circ}$. Table I shows the comparisons between calculated and measured quantities for the various $n \nu_{7}^{l}$ states which have been analyzed. The molecular constants for the ground and $2 \nu_{7}{ }^{0}$ states are from Ref. (1), while those for the $\nu_{7}^{1}$ and $2 \nu_{7}^{2}$ states are from Ref. (2). The latter values differ slightly from those previously reported since the data have subsequently been reanalyzed using lower-level combination differences only, and improved measurements for the $P$-branch lines associated with the $2 \nu_{7}^{2}$ states have been included. The analysis of the remaining states will be reported in a separate publication (19). The uncertainties in the computed quantities are in all cases much less than the experimental uncertainties.

The only systematic discrepancy between theory and experiment shown in Table I is that the predicted $B^{\prime \prime}$ values for most states are slightly high. The biggest errors are roughly $1.3 \times 10^{-4} \mathrm{~cm}^{-1}$ for the $\nu_{7}{ }^{1}$ states and $2.0 \times 10^{-4} \mathrm{~cm}^{-1}$ for the $3 \nu_{7}{ }^{1}$ states, and in both cases they are substantially greater than the estimated experimental uncertainty of $\pm 3 \times 10^{-5} \mathrm{~cm}^{-1}$. On the other hand, the $l$-doubling constants $q_{\imath}{ }^{\prime \prime}$ are predicted quite well for both levels.

We show in Fig. 2 a comparison between the computed and measured combination differences for the ground and $2 \nu_{7}{ }^{0}$ states. The ground-state data show much less scatter since they were obtained by averaging the results of 6 different bands having a common lower level (1). The solid lines give the computed results. A least-squares fit to the equat- 


\begin{tabular}{|c|c|c|c|c|}
\hline State & Energy ${ }^{d}$ & $B^{\prime \prime}$ & $D^{11}\left(10^{-8}\right)$ & $8_{\ell}^{11}\left(10^{-4}\right)$ \\
\hline $0^{\circ}$ & 0 & $0.07556255^{\mathrm{a}}$ & $\begin{array}{l}3.747 \\
(3.817 \pm 0.04)^{c}\end{array}$ & \multirow{4}{*}{$\begin{array}{c}4.11 \\
(4.38)\end{array}$} \\
\hline$v_{7}^{1 \mathrm{cc}}$ & $\begin{array}{r}18.24 \\
(18.5)\end{array}$ & $\begin{array}{l}0.076153 \\
(0.076012\rangle\end{array}$ & $\begin{array}{l}5.4 \\
(4.4 \pm 0.31)\end{array}$ & \\
\hline$v_{7}^{1 d d}$ & $\begin{array}{r}18.24 \\
(18.5)\end{array}$ & $\begin{array}{l}0.076564 \\
(0.076450)\end{array}$ & $\begin{array}{l}7.3 \\
(6.0 \pm 1.1)\end{array}$ & \\
\hline $2 v_{7}^{2}$ & $\begin{array}{r}45.46 \\
(46.2)\end{array}$ & $\begin{array}{l}0.076990 \\
(0.076994)^{b}\end{array}$ & $\frac{5.9}{(6.2 \pm 0.5)^{b}}$ & \\
\hline $2 v_{7}^{0}$ & $\begin{array}{l}60.7022^{a} \\
(60.7)\end{array}$ & $0.0762799^{3}$ & $\begin{array}{l}1.96 \\
(1.760 \pm 0.416)^{c}\end{array}$ & \multirow{5}{*}{$\begin{array}{l}5.31 \\
(5.29)\end{array}$} \\
\hline $3 v_{7}^{3}$ & $\begin{array}{r}79.61 \\
(80.3)\end{array}$ & $\begin{array}{l}0.077584 \\
(0.077515)^{b}\end{array}$ & $\begin{array}{l}5.6 \\
(2.1 \pm 3.0)\end{array}$ & \\
\hline $3 v_{7}^{1 c}$ & $\begin{array}{r}98.53 \\
(97.9)\end{array}$ & $\begin{array}{l}0.076821 \\
(0.076616)\end{array}$ & $\begin{array}{l}2.8 \\
(0.82 \pm 0.6)\end{array}$ & \\
\hline $3 v_{7}^{10}$ & $\begin{array}{r}98.53 \\
(97.9)\end{array}$ & $\begin{array}{l}0.077350 \\
(0.077145)\end{array}$ & $\begin{array}{l}4.1 \\
(1.57 \pm 0.6)\end{array}$ & \\
\hline $4 v_{7}^{0}$ & $\begin{array}{r}144.17 \\
(144.7)\end{array}$ & $\begin{array}{l}0.077381 \\
(0.077374)\end{array}$ & $\begin{array}{l}2.9 \\
(7.9 \pm 3.5)\end{array}$ & \\
\hline
\end{tabular}

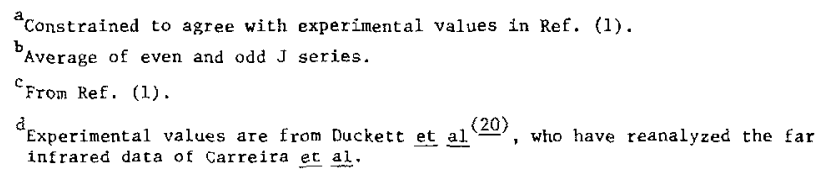

tion

$$
\Delta_{2} F(J) /(4 J+2)=B_{\nu}-2 D_{\nu}\left(J+\frac{1}{2}\right)^{2}+3 H_{\nu}\left(J+\frac{1}{2}\right)^{4},
$$

using the computed ground-state combination differences yields $D_{\nu}=3.747 \times 10^{-8}$ $\mathrm{cm}^{-1}$ and $H_{\nu}=5.94 \times 10^{-13} \mathrm{~cm}^{-1}$. These values are in excellent agreement with the results $D_{\nu}=3.817 \times 10^{-8} \mathrm{~cm}^{-1}$ and $H_{y}=6.019 \times 10^{-13} \mathrm{~cm}^{-1}$, obtained by fitting the expcrimental data in a similar manner (1).

Figure 2 provides convincing evidence that the basic assumption of our model, namely the neglect of all other vibrations, is valid. The agreement between observed and predicted $D$-terms for the ground state is actually better than we should expect. A linear molecule will generally have a $D$ term of magnitude $4 B_{0}{ }^{3} / \omega_{\mathrm{s}}{ }^{2} \approx 2.5 \times 10^{-9}$ $\mathrm{cm}^{-1}$, where $\omega_{\mathrm{s}}$ is the lowest symmetric stretching frequency. Assuming that centrifugal distortion effects are additive, then the predicted $D$ term should be approximately $2.5 \times 10^{-9} \mathrm{~cm}^{-1}$ low. This discrepancy has the same sign, but it is $3-4$ times larger than the observed discrepancy of $0.7 \times 10^{-9} \mathrm{~cm}^{-1}$.

Table II shows the lowest 6 Raman transitions reported by Carreira et al. (8) compared with our predicted results. The agreement is very good, especially considering the fact that our fitting procedure involves only the first transition. We have made no attempt to fit the far-infrared data by the same authors. The highly anharmonic potential leads to a rather simple Raman spectrum, and the assignments of at least the first few lines appear to be straightforward. In contrast, the far infrared spectrum is much more complex, and the assignments of even the first few features are somewhat 


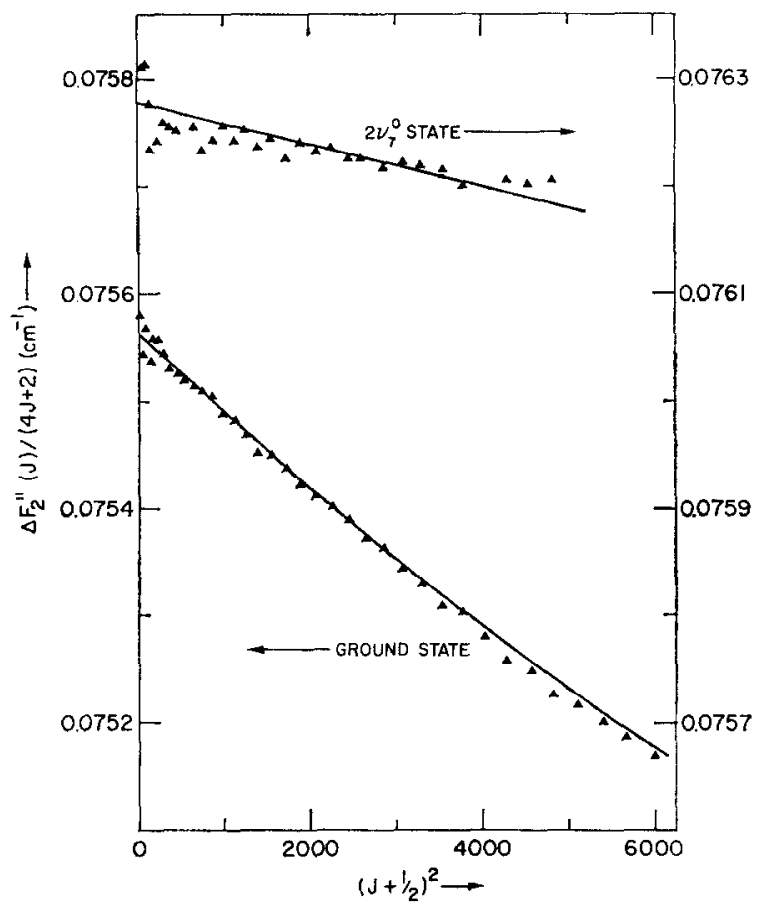

FIG. 2. Comparison between experimental and theoretical combination differences for the ground and $2 \nu_{7}{ }^{\mathrm{l}}$ state of $\mathrm{C}_{3} \mathrm{O}_{2}$. The data points are from Ref. (1), while the solid curves are computed using the model described in the text.

questionable. In fact, Duckett, Mills, and Robiette (20) have recently reassigned the far-infrared spectra from Ref. (8), and their values for the $\nu_{7}$ energy levels, which are shown in Table $I$, are in good agreement with our predictions.

Table II. Comparison between observed and calculated Raman frequencies

for $\mathrm{C}_{3} \mathrm{O}_{2}$. The experimental data are froju Ref. ( $(8)$.

\begin{tabular}{ccc}
\hline Transition & Observed $\left(\mathrm{cm}^{-1}\right)$ & Calculated $\left(\mathrm{cm}^{-1}\right)$ \\
\hline $2^{0}+0^{0}$ & 61.0 & $60.70^{\mathrm{a}}$ \\
$3^{1}+1^{1}$ & 79.4 & 80.29 \\
$4^{0}+2^{0}$ & 84.0 & 84.10 \\
$4^{2}+2^{2}$ & 91.5 & 91.32 \\
$5^{1}+3^{1}$ & 94.9 & 95.74 \\
$5^{3}+3^{3}$ & 101.0 & 100.53 \\
\hline
\end{tabular}




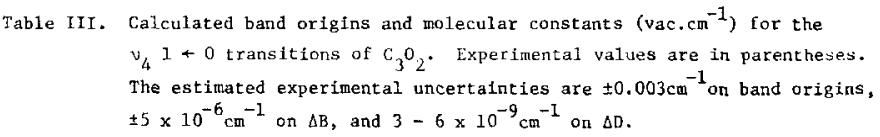

\begin{tabular}{|c|c|c|c|c|}
\hline Transition & Band Origin & $B^{\prime}-B^{n}\left(10^{-4}\right)$ & $D^{+}-D^{\prime \prime}\left(10^{-8}\right)$ & $q_{2}^{5}-q_{2}^{n}\left(10^{-4}\right)$ \\
\hline$v_{4}+0^{o}$ & $(1587.3905)$ & $6.432^{\mathrm{a}}$ & $\begin{array}{l}1.89 \\
(1.86)\end{array}$ & \multirow{4}{*}{$\begin{array}{c}1.02 \\
(0.89)\end{array}$} \\
\hline$v_{4}+v_{7}^{1 c}+v_{7}^{1 c}$ & $\begin{array}{l}1580.902 \\
(1580.896)\end{array}$ & $\begin{array}{c}4.13 \\
(4.46)\end{array}$ & $\begin{array}{l}2.04 \\
(2.0)\end{array}$ & \\
\hline$v_{4}+v_{7}^{1 d}+v_{7}^{1 d}$ & $\begin{array}{l}1580.902 \\
(1580.900)\end{array}$ & $\begin{array}{c}5.15 \\
(5.35)\end{array}$ & $\begin{array}{l}2.76 \\
(1.4)\end{array}$ & \\
\hline$v_{4}+2 v_{7}^{2}-2 v_{7}^{2}$ & $\begin{array}{l}1575.348 \\
(1575.093)^{b}\end{array}$ & $\frac{4.11}{(3.96)^{b}}$ & ${ }_{(0.11)^{b}}$ & \\
\hline$v_{4}+2 v_{7}^{0}+2 v_{7}^{0}$ & $1583.4300^{\mathrm{a}}$ & $-0.119^{a}$ & $\begin{array}{l}1.1 \\
\{0.263\}\end{array}$ & \multirow{5}{*}{$\begin{array}{c}0.58 \\
(0.55)\end{array}$} \\
\hline$v_{4}+3 v_{7}^{3}+3 v_{7}^{3}$ & $\begin{array}{l}1570.012 \\
(1569.503)^{b}\end{array}$ & $(4.14)^{3.80}$ & $\begin{array}{l}1.64 \\
(0.94)^{b}\end{array}$ & \\
\hline$\nu_{4}+3 v_{7}^{1 c}+3 v_{7}^{1 c}$ & $\begin{array}{l}1575.331 \\
(1575.996)\end{array}$ & $\begin{array}{l}1.32 \\
(1.26)\end{array}$ & $\begin{array}{c}1.3 \\
(0.19)\end{array}$ & \\
\hline$v_{4}+3 v_{7}^{1 d}+3 v_{7}^{1 d}$ & $\begin{array}{l}1575.331 \\
(1575.996)\end{array}$ & $\begin{array}{c}1.90 \\
(1.81)\end{array}$ & $\begin{array}{l}1.4 \\
(0.28)\end{array}$ & \\
\hline$v_{4}+4 v_{7}^{\circ}+4 v_{7}^{0}$ & $\begin{array}{l}1572.990 \\
(1572.976)\end{array}$ & $\begin{array}{l}0.31 \\
(0.25)\end{array}$ & $\begin{array}{l}-0.58 \\
(-0.002)\end{array}$ & \\
\hline
\end{tabular}

${ }^{a}$ Constrained to agree with experiment.

berage of even and odd $J$ series.

\section{B. Stretching Modes Excited Simultaneously with $\nu_{7}$}

The model used to treat the $n \nu_{7}{ }^{l}$ states should be equally valid for states of the type $y_{s}+n \nu_{7}{ }^{l}$, where $\nu_{s}$ is a high-frequency stretching vibration. The effect of the highfrequency mode will be to produce small changes in the parameters $B_{0}, V_{2}$, and $V_{4}$. The most extensive measurements on states of this type have been done on the $\nu_{4} 1 \leftarrow 0$ band and the various $\nu_{7}$ hot bands associated with it. This vibration is the asymmetric $\mathrm{C}=\mathrm{C}$ stretching mode at about $1587 \mathrm{~cm}^{-1}$. Since the energies and $B$ values of the ground and $2 \nu_{7}{ }^{0}$ states are known, measurements of the $\Delta B$ values for the transitions $\nu_{4} \leftarrow 0^{0}$ and $\nu_{4}+2 \nu_{7}{ }^{0} \leftarrow 2 \nu_{7}^{0}$ along with a measurement of the shift of the $2 \nu_{7}{ }^{0}$ hot band allow us to use exactly the same fitting procedure as in the previous section.

The parameters which result for the $\nu_{4}$ state are $B_{0}=0.0733140 \mathrm{~cm}^{-1}, V_{2}=-2228$ $\mathrm{cm}^{-1}$, and $V_{4}=21934 \mathrm{~cm}^{-1}$. The potential has a $56.58 \mathrm{~cm}^{-1}$ barrier at $\alpha=0$ with a minimum at $\alpha=13.02^{\circ}$. The effect of exciting $\nu_{4}$ is thus to increase the barrier by nearly a factor of two and to shift the minimum by $2^{\circ}$. The quartic term in the potential changes only slightly.

Table III shows the comparisons between calculated and measured quantities for nine $\nu_{4}+n \nu_{7}{ }^{l}$ states. The data for the first five of these are obtained by using a leastsquares fit to the line positions reported in Ref. (2), keeping the lower-level constants constrained to be those given by the experimental numbers in Table I. A different assignment for the $2 \nu_{7}{ }^{0}$ band origin has been used. The reason for choosing this new assignment and the analysis for the remaining states will be reported later (19). The 
agreement is again remarkably good, the predicted band origins and $\Delta B$ values agreeing with the measured quantities to within $\pm 0.7 \mathrm{~cm}^{-1}$ and $\pm 3 \times 10^{-5} \mathrm{~cm}^{-1}$, respectively.

Of the states studied by Mantz et al. (1) the only one for which there are sufficient data to use the same fitting method to determine the $\nu_{7}$ potential is $\nu_{2}+\nu_{3}$. The parameters we find for this state are $B_{0}=0.0731114 \mathrm{~cm}^{-1}, V_{2}=-1631 \mathrm{~cm}^{-1}$, and $V_{4}=24294$ $\mathrm{cm}^{-1}$. The potential has a $27.36 \mathrm{~cm}^{-1}$ barrier with a minimum at $10.56^{\circ}$. The barrier is slightly lower than it is in the ground state and the quartic term is larger. This means that the hot bands of $\nu_{2}+\nu_{3}$ will all be shifted to higher frequency in contrast to the lower-frequency shifts observed for $\nu_{4}$. The only comparisons between theory and experiment which we can make for the $\nu_{2}+\nu_{3}$ states are for the $\Delta D$ terms. The predicted $\Delta D$ value for the $\nu_{2}+\nu_{3} \leftarrow 0$ transition is in excellent agreement with experiment, while that for the $\nu_{2}+\nu_{3}+2 \nu_{7}^{0} \leftarrow 0$ has the right sign but is nearly a factor of 30 smaller in magnitude than the experimental value.

Figure 3 shows the $\nu_{7}$ potential and energy levels for the ground, $\nu_{4}$, and $\nu_{2}+\nu_{3}$ states. The changes in the energy levels in this figure can be associated directly with the linear to bent transitions discussed by Thorson and Nakagawa for molecules of this type. Comparing the energy levels in Fig. 3 with those in the correlation diagram in Fig. 2 of Ref. (10), we see that the $\nu_{4}$ state corresponds to a more bent configuration, the $\nu_{2}+\nu_{3}$ state to a more linear configuration, and the ground state is intermediate.

In linear molecules the excitation of a stretching vibration usually leads to $\Delta B$ values which are negative (21). The apparently anomalous signs of $\Delta B$ for most of the

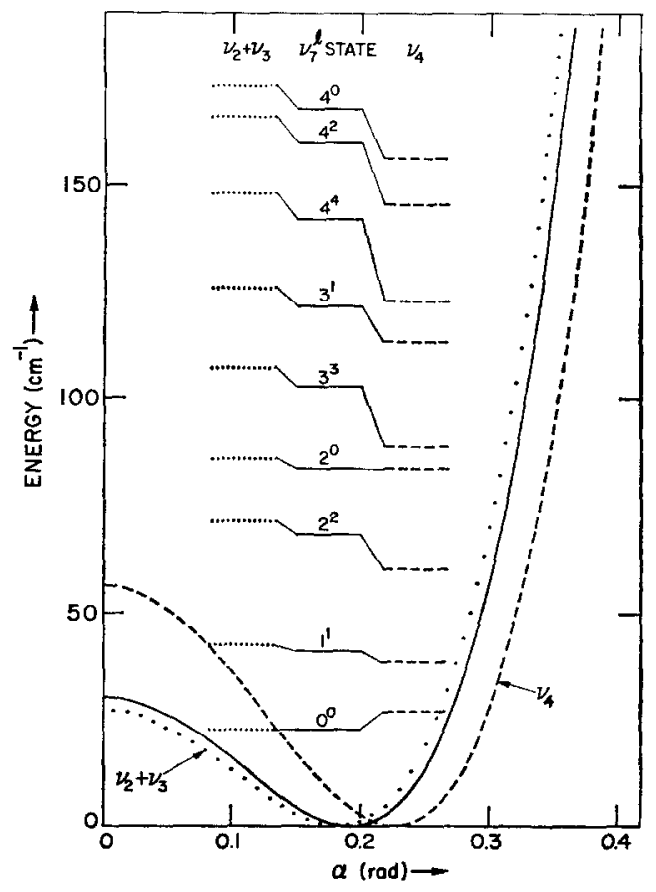

FIG. 3. Calculated energy levels and potential function for the $\mathrm{C}_{3} \mathrm{O}_{2} \nu_{2}$ bending mode in the ground state (solid lines), the $\nu_{4}$ state (dashed lines), and the $\nu_{2}+\nu_{3}$ state (clotted lines). The zero of the energy scale is arbitrarily chosen to coincide with the potential minima. 
transitions in Table III arise entirely from the change in the $\nu_{7}$ potential. The direct change in the rotational constant produced by the $\nu_{4}$ excitation is given by $\Delta B_{0}=-1.998$ $\times 10^{-4} \mathrm{~cm}^{-1}$, which has the usual negative sign. The experimental $\Delta B$ values result from the combined effects of the change in $B_{0}$, which gives a negative contribution, and the change in the $\nu_{7}$ potential, which gives a positive contribution. In every case except $2 \nu_{7}{ }^{0}$ the positive contribution dominates.

The upper-state levels of several near-infrared bands of $\mathrm{C}_{3} \mathrm{O}_{2}$ have been tentatively assigned by Mantz et al. ( 1 ) on the basis of the observed $\Delta B$ values. If these bands have anomalous $\Delta B$ values, as is the case for $\nu_{4}$, then several different assignments appear more likely. For example, the upper state of the band at $3774.462 \mathrm{~cm}^{-1}$, assigned as $\nu_{4}+4 \nu_{6}{ }^{0}$, could be $\nu_{1}+\nu_{4}$. The upper state of the band at $4471.8375 \mathrm{~cm}^{-1}$, assigned as $\nu_{3}+4 \nu_{6}^{0}$, could be $\nu_{1}+\nu_{3}$. Finally, the upper state of the weak band at 3830.6023 $\mathrm{cm}^{-1}$, assigned as $\nu_{4}+2 \nu_{5}^{0}+2 \nu_{8}^{0}$, could be $\nu_{1}+\nu_{4}+2 \nu_{7}{ }^{0}$. After a few more of the corresponding hot bands have been analyzed in this spectral region, the consistency of the upper-state assignments can be readily checked by applying the same fitting procedure we have used for the $\nu_{4}$ band.

\section{ACKNOWLEDGMENTS}

We wish to thank C. W. Peters for much encouragement and advice. We also thank I. M. Mills for sending us a copy of Ref. (20) prior to publication.

Received : May 24, 1976

\section{REFERENCES}

1. A. W. Mantz, P. Connes, G. Guelachvili, and C. Amiot, J. Mol. Spectrosc. 54, 43 (1975).

2. W. H. Weber, P. D. Maker, and C. W. Peters, J. Chem. Phys. 64, 2149 (1976).

3. J. R. SAbin and H. Kim, J. Chem. Phys. 56, 2195 (1972).

4. A. Almenningen, S. P. Arnesen, O. Bastiansen, H. M. Seip, and R. Seip, Chem. Phys. Letl. 1, 569 (1968).

5. A. Clark and H. M. Seip, Chem. Phys. Lett. 6, 452 (1970). See also M. Tanimoto, K. Kuchitsu, AND Y. Morino, Bull. Chem. Soc. Japan 43, 2776 (1970).

6. R. L. Redington, Spectrochim. Acta A 23, 1863 (1967).

7. W. H. Simth and G. E. Lerol, J. Chem. Phys. 45, 1767 (1966).

8. L. A. Carreira, R. O. Carter, J. R. Durig, R. C. Lord, and C. C. Milionis, J. Chem. Phys. 59, 1028 (1973).

9. J. T. Hougen, P. R. Bunker, and J. W. C. Johns, J. Mol. Spectrose. 34, 136 (1970).

10. W. R. Thorson and I. Nakagawa, J. Chem. Phys. 33, 994 (1960).

11. H. Goldstern, "Classical Mechanics," p. 134. Addison-Wesley, Cambridge, Mass., 1950.

12. See for example K. S. Cheng, J. Math. Phys. 13, 1723 (1972).

13. H. H. Nielsen, "Handbook of Physics," Vol. 37-1, Section 13, p. 173. Springer-Verlag, Berlin, 1959.

14. See Section 23, Ref. (13).

15. See Section 9, Ref. (13).

16. See Section 42, Ref. (13).

17. G. BIRKHOFF ANd G.-C. Rota, "Ordinary Differential Equations," Chap X. Ginn, Boston, 1962.

18. See Ref. (17), Chap. VIII.

19. C. W. Peters, W. H. Weber, ANd P. D. Maker, to appear.

20. J. A. Duckett, I. M. Mills, ANd A. G. Robiette, J. Mol. Spectrosc. 63, 249 (1976); and I. M. MILls, private communication.

21. G. Herzberg, "Infrared and Raman Spectra," p. 370. VanNostrand-Reinhold, New York, 1945. 